\title{
A bayesian game-theoretic demand response model for the smart grid
}

\author{
Ulas Baran Baloglua ${ }^{\mathrm{a}}$, Yakup Demir ${ }^{\mathrm{b}}$ * \\ ${ }^{a}$ Engineering Faculty, Tunceli University, Tunceli 62000, Turkey \\ ${ }^{b}$ Electrical and Electronics Engineering Department, Firat University, Elazig 23119, Turkey
}

\begin{abstract}
Smart grids are considered to replace traditional grids by adopting advanced technologies. Peak amounts would be an important future problem because of new devices such as electric cars, smart home appliances and the others with high-energy consumptions. In a smart grid environment, these problems should be eliminated. As a solution a management and control mechanism is required. In a smart grid, demand-side management or demand response system aims to obtain an evenly distributed consumption. In this study, a demand response model is proposed. This model uses game-theoretical methodologies with a dynamic pricing scheme. In an intelligent system of consumers and the utility company, energy prices are dynamic. Lowering prices may motivate consumers to shift some of their daily optional consumption. The motivation for saving on energy bills can be used with game-theoretical methodologies to construct a solution that eliminates peak amounts.
\end{abstract}

Keywords: Demand response model, dynamic pricing, game theory, smart grid

\section{Introduction}

In the next decade, smart grids are expected to replace the traditional grids. Advancing information technologies enable grids to transmit power in a more efficient way than they do before. Grids are also starting to have new adaptation mechanisms to frequently changing demand and supply conditions. A smart grid should have a demand-side management or a demand response model to construct such an efficient and robust system.

Consumers are now using new devices such as electric cars and appliances for smart homes. As a result, the behavior of electrical energy consumption has been dramatically changing. In a grid, the preferred consumption type is homogenously distributed consumption over a 24-hour period with no or minimum peak amounts. The main motivation of a demand response model is to achieve this goal, and it can be described as controlling energy consumption thru metering devices of consumers.

There are previous studies in the literature, which proposed solutions to make grids more efficient and robust. Some of these studies used agent-based computational methods to model a demand-side management [1], [2]. In several studies, demand-side management was modelled by using gametheoretical methodologies [3]-[7]. Most of the demand-side management methods used in the literature especially focused on computational techniques rather than modelling the consumer behavior in a more lifelike way, where this study separates from previous studies. The proposed model adds flexibility by using Bayesian game theory and by taking account of unpredictable consumer behaviors.

Smart metering devices are required to compose a demand response model. These devices can be used in decentralized and centralized environments to obtain a bidirectional communication between consumers and the utility. This communication helps utility to collect the usage data, which is essential for all of the demand response models. Smart metering devices are another research area, but they are

\footnotetext{
* Manuscript received June 1, 2015; revised July 23, 2015.

Corresponding author: Tel.: +905426924226; E-mail address: baloglu @ tunceli.edu.tr.

doi: $10.12720 /$ sgce.4.2.132-138
} 
essential to understand how a demand response model works. For metering device communication, different technologies can be used such as cellular networks, sensor networks, ZigBee network, cognitive radio network or McWill network [8]. These technologies should provide fast, reliable and secure communication; otherwise it would be impossible to add intelligence to smart grids.

In this paper, a demand response model is proposed. This model uses game-theoretical methodologies and a dynamic pricing scheme to achieve an evenly distributed daily load in the smart grid. The rest of the paper is organized as follows. Proposed demand response model and dynamic pricing scheme are explained in the second section of the paper. In the third section, the problem definition is given by using game-theoretical methodologies. Simulation results are given in the fourth section. Finally, the conclusion is given in the fifth section.

\section{Proposed Model}

In many countries same institutions transmit and distribute the electricity; there is also a tendency for privatization of these institutions [3]. These institutions have a common goal to cut back on costs, efficiently integrate new technologies and to develop environmental friendly green technologies. Further, market liberalization and changing regulations lead countries to redefine their electric energy systems [9]. Reducing peak amounts is an important goal for these future electric systems. Either consumption should be lowered or it should be intelligently distributed to reduce peak amounts. An efficient demand response model encourages consumers to reschedule some of their appliances.

\subsection{Smart grids}

Smart grids differ from the traditional grids by having advanced sensors and smart metering devices, and they differ by using communication, information, and control technologies [10]. A smart grid is more efficient, reliable, renewable, reconfigurable and secure than a traditional grid. It has strategies to response any events that occur at any point of the grid from power generation to transmission and from distribution to consumption [11].

In a smart grid, demand response is an important mechanism for reliability and efficiency. A bidirectional communication mechanism is required to construct a demand response model. This communication mechanism can be either between the consumers or between the consumers and the utility company. As a result, smart metering devices are in the heart of demand response models, and they are one of the key components in a smart grid. These metering devices should comply with necessary specifications to provide the required bidirectional communication.

\subsection{Game theory}

Game theory analyzes strategic behaviors of rational agents [12]. In the game theory, there is a finite set of players who determine their strategy to achieve a common goal. With every new step taken, strategies of the players will change. Finally, there will be a point where none of the players can change its strategy; this point is the Nash Equilibrium. Reaching this common point will satisfy goals of every player in the system. Nash Equilibrium produces an optimal solution for a problem.

Game theory is a universal methodology, which applied in various fields from the economy to the biology. Advanced technologies and services of a smart grid make it benefits from game-theoretical methodologies about its design and analysis [13].

In the game theory, games are classified as non-cooperative and cooperative games [14]. In the noncooperative games, players determine their strategies for conflicting interests. A communication mechanism may exist, but it doesn't provide information about strategies of other players. There are two types of non-cooperative games. First one is known as static games, and the other one is dynamic games. Dynamic games differ by enabling players to act more than once in a game. On the contrary, in static games players act only once. In cooperative games, players communicate and negotiate with each other to maximize their benefits. 
Congestion game is a specific game type, where there are two sets; one is for the players, and the other one is for the resources. For every player in the game, there is a separate finite strategy set. Those strategy sets are used to allocate the resources. Since resource allocation has a cost, every player tries to find its best strategy to minimize that cost. At the end of the game, combining all individual strategy sets will form a solution set. In a Bayesian game, there is incomplete information such that one player does not have the knowledge of other player's payoffs.

\subsection{Demand response model}

A demand response model can receive data from the consumers and also can send a response to them. Consumers should communicate with each other to achieve a common goal. Thus, there should be a bidirectional communication mechanism between the consumers and between the consumers and the utility. The utility updates its prices according to grid load data calculated from consumption; it also provides recent price information to its consumers.

In Fig. 1, a demand response model is given. In this model, there are $n$ consumers, which are denoted as $C 1 \ldots C n$, and there is a utility company, which is denoted as $U C$. In the figure, bidirectional arrows show the data exchange between the consumers and the utility company. The energy price data and the consumption data are transmitted.

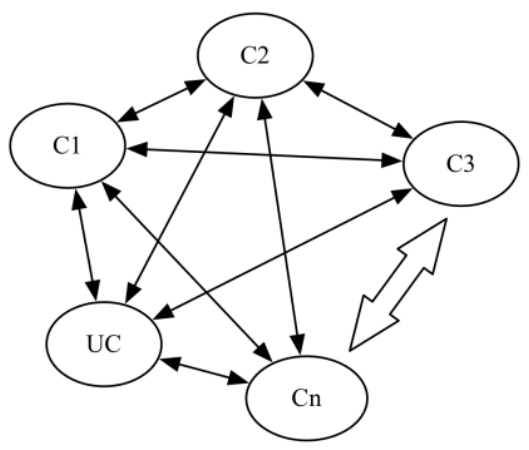

Fig. 1. Demand response model.

An effective demand response model is beneficial for both consumers and the utility company. In the proposed model, there is a common goal of the utility company and the consumers; this goal is to reduce peak amounts in the grid by evenly distributing the daily load. The game-theoretical methodology with a dynamic pricing scheme may generate equilibrium because utility company's pricing strategy combined with the strategies of the consumers who would try to lower their bills.

\subsection{Pricing scheme}

There are numerous pricing schemes applied to smart grids such as flat pricing, peak load pricing and dynamic pricing [15]. In flat pricing, the utility company determines a fixed price for all time intervals. This fixed price is determined in the beginning, and it won't change during the operation. Peak load pricing differs by determining different pricing for different time intervals. Contrarily, in dynamic pricing there is no price fixing and the price is calculated separately for each time interval. The main advantage of dynamic pricing is a company delays its pricing decision until the market conditions are known so that the company can adjust prices, according to its strategy.

In the proposed model, dynamic price calculation requires data of consumption amount for each time interval. There should be a penalty for hours with high consumption, and hours with low consumption should have a reward as well. Rewards have limitations to prevent a pricing, which is lower than the production cost. Penalties also shouldn't produce an unacceptable very high pricing. Set of proper prices is defined to prevent these undesirable outcomes. 
Let $\rho=\left[\rho_{\min }, \rho_{\max }\right]$ is the set of the proper prices that are determined by the utility company. This pricing function is lower and upper bounded. Between these bounds there exists a one-to-one relationship. For the proposed model, dynamic pricing scheme takes the following form:

$$
F(\rho, L)=\left\{\begin{aligned}
\rho_{\min } & L & \leq L_{\min } \\
r \sigma \frac{A+L}{A} & \mathrm{~L}_{\min } & <L<L_{\max } \\
\rho_{\max } & L & \geq L_{\max }
\end{aligned}\right.
$$

where, $\rho$ is the set of prices, $r \in \rho$ is the price determined by the utility company, $L$ is the load values of the utility, $A$ is a positive constant and $\sigma$ is the pricing strategy of the utility company. The price is increasing function of loads. The utility company can scale the rewards and the penalties with the pricing strategy parameter.

\section{Problem Definition}

In this section, the problem definition is given mathematically by using game-theoretical methodologies. In a demand response model, the main problem is defined as balancing the load in the most suitable way. The problem is not reducing energy usage but balancing the load, and this can be achieved by intelligently shifting energy consumption.

Let there are $n$ players (consumers) in a smart grid. For each of the player, total daily power consumption is divided into a total of $m \tau$-minutes intervals. In this situation, the daily power consumption of the player $i$ is defined with a vector,

$$
t_{i}=\left[t_{i}^{1}, \ldots t_{i}^{m}\right], 1 \leq i \leq n
$$

where, for every player $i$ its $\mathbf{t}_{\mathbf{i}}$ vector is divided into a total of $m \tau$-minutes intervals. The utility company is accepted as a player so that the total number of players becomes $n+1$. Let strategy set of each player $i$ is given by the following equation,

$$
S_{i}, 1 \leq i \leq n+1
$$

where, $S_{i}$ is the strategy set of player $i$ and $S_{n+1}$ is the strategy set of the utility company. Strategies of all players are collected in the set $N$ as,

$$
N=\left(S_{1}, S_{2}, \ldots, S_{n}, S_{n+1}\right)
$$

For each player $i$, related goal function is to minimize

$$
\sum_{k=1}^{m} t_{i}^{k}, 1 \leq i \leq n
$$

where, $t_{i}^{k}$ is the power consumption of $i^{\text {th }}$ player in the $k^{\text {th }}$ time interval. In real life situations, some consumers may not bother with shifting their loads. As a result, in the simulation environment a selection criterion is used to distinguish such consumers. This selection criterion also helps the model to escape from Avalanche effects. Avalanche effects are peaks caused by demand response models in which all consumers use electricity at the same time when unit pricing is low.

For the utility company, the goal will have an instant load closer to the average load. The average load is defined as follows.

$$
1 / m\left[\sum_{i=1}^{n} \sum_{k=1}^{m} t_{i}^{k}\right], 1 \leq i \leq n
$$


Finally, to address the incomplete information in utility company's cost function the game is described as a Bayesian game,

$$
G=\left(N, \Omega, A_{i}, T_{i}, S_{i}, P_{i}\right), 1 \leq i \leq n+1
$$

where, $N$ is the finite set of players, $\Omega$ is the finite set of states of nature, $A_{i}$ denotes the action set, $T_{i}$ denotes the type of player $i, S_{i}$ denotes $i^{\text {th }}$ player's strategy set and finally $P_{i}$ denotes the common probability function of player $i$.

We assume that a consumer's cost function has the form $\Omega_{i}(c)$, where $c$ is a common cost for all consumers. The objective of each consumer is to consume while the pricing is low. Payoff function set is defined as $U=\left\{u_{1}, u_{2}, \ldots u_{n+l}\right\}$ where $u_{i}$ is defined as,

$$
u_{i}: A_{1} \times A_{2} \times \ldots \times A_{n+1} \times T_{1} \times T_{2} \times \ldots \times T_{n+1} \rightarrow R
$$

For every time step, some consumers dynamically react to the pricing information, which is calculated and transmitted by the utility company. We apply the Bayesian game model to every step with dynamically adjusted new unit prices. This multi-step Bayesian game is continuously used to update beliefs of consumers.

\section{Simulation Results}

A simulation environment was constructed to test the proposed demand response model. We evaluate the proposed model in terms of load and game steps. In the simulation environment, there are 2000 consumers and each of them has two types of consuming demand. First consuming demand type is demands that cannot be shifted. For example, consumers do not shift their lighting consumption to another time interval or they do not turn off their television sets to lower their bills. The second type is demands that can be shifted. A consumer may charge an electric car in another time interval unless there isn't any emergency situation, or they may postpone operating their dishwashers.

In the environment, consumers use appliances that are given in Table 1. The consuming behavior of the virtual population is calculated according to this data. In the simulation environment, each household is a player of the game, and they behave independently. Furthermore, each household has randomly selected appliances and for every appliance in a household, demands for usage are randomly determined.

\begin{tabular}{|c|c|c|c|}
\hline Appliance Type & $\begin{array}{l}\text { Consumption } \\
(\mathrm{kWh})\end{array}$ & $\begin{array}{l}\text { Average Working } \\
\text { Hour(s) }\end{array}$ & Necessity $(\mathrm{Yes} / \mathrm{No})$ \\
\hline Refrigerator & 0.15 & 24 & Yes \\
\hline Dish Washer & 2.00 & 2 & No \\
\hline Washing Machine & 2.50 & 2 & No \\
\hline Oven & 2.00 & 2 & No \\
\hline Electric Car (EV) & 9.70 & 2 & No \\
\hline Television & 0.20 & 10 & Yes \\
\hline Iron & 1.80 & 1 & No \\
\hline Kettle & 1.50 & 1 & No \\
\hline Satellite Receiver & 0.05 & 10 & Yes \\
\hline Telephone & 0.01 & 24 & Yes \\
\hline Desktop Computer & 0.45 & 6 & No \\
\hline Air Conditioner & 2.00 & 10 & Yes \\
\hline Lighting & 0.40 & 7 & Yes \\
\hline
\end{tabular}

Table 1. Appliances in the simulation environment

In the Fig. 2 total consumption values over the time intervals are given. This figure displays data before and after the application of demand response model. It is assumed that utility company's pricing strategy parameter is selected from the interval $(0,1]$ and this parameter remains fixed during the simulation. For each time interval, dynamic pricing value is calculated with the formula that is given in (1). The parameter $\tau$ is selected as 30 and grid loads are calculated separately for each time interval. 


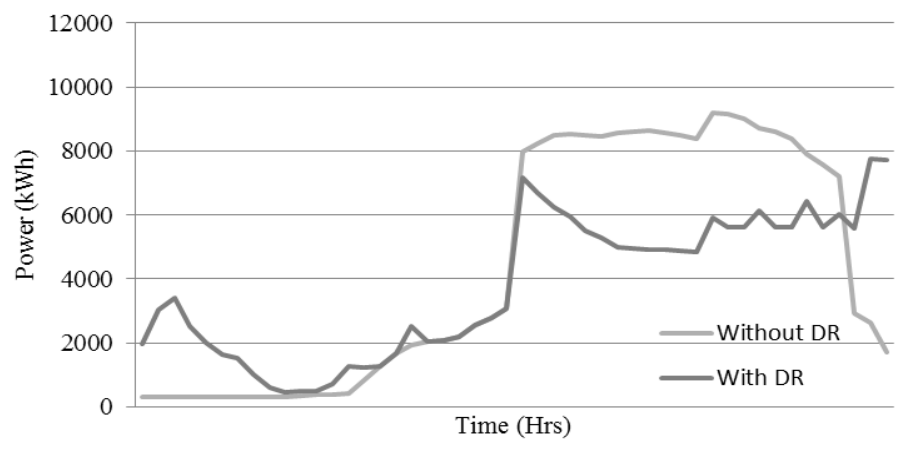

Fig. 2. Total consumption values over time after applying demand response (DR) model.

As it is seen in the figure, after applying the demand response model consumers shifted some of their optional consuming demands to the most suitable time intervals according to their game strategies. Additionally, it can be observed that peak load decreased with demand response.

Fig. 3 shows total consumption values after the demand response model was applied. This figure illustrates consumption values over time for a different number of Bayesian game steps. Learning process is an internal part of a game theoretic model. As shown in the figure, increasing the number of Bayesian game steps produces better results. This result is expected since every game step is a part of a learning process, and it helps consumers to improve their belief sets.

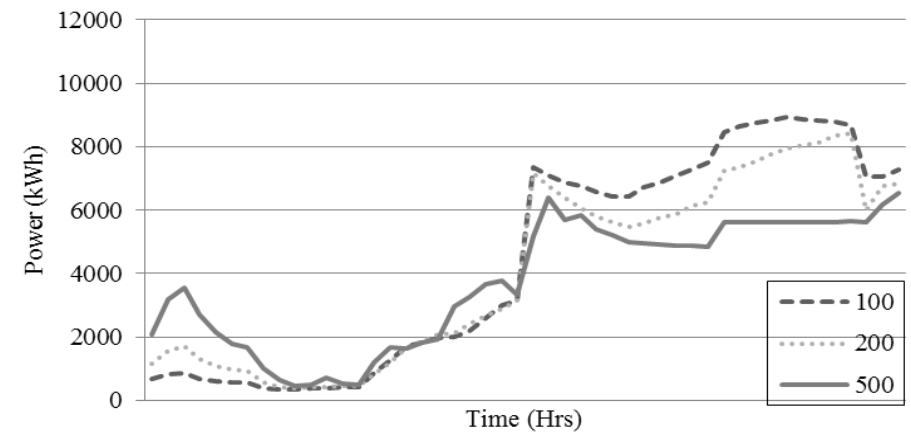

Fig. 3. Demand response with different number of Bayesian game steps.

\section{Conclusion}

In this study, a demand response model with a dynamic pricing scheme is proposed. The proposed model uses game-theoretical methodologies and applies a dynamic pricing scheme. From the utility company's point of view, demand response model helps reducing peak amounts in a grid. Preventing peaks provide crucial benefits such as decreasing operation costs and lowering carbon emission levels. From the consumers' point of view, a demand response model helps them to reduce their bills by only shifting some of their optional consumption. For these different goals, a problem is defined by using Bayesian game, and a model is constructed.

Simulation results demonstrate that the proposed model evenly distributes the daily load in smart grid and prevents Avalanche effects. It is also observed that increasing Bayesian game steps improve the learning process.

\section{References}

[1] Ramchurn SD, Vytelingum P, Rogers A, Jennings N. Agent-based control for decentralised demand side management in the 
smart grid. In: Proc. of 10th Int. Conf. on Autonomous Agents and Multiagent Systems - Innovative Applications Track, 2011:5-12.

[2] Logenthiran T, Srinivasan D, Shun TZ. Multi-agent system for demand side management in smart grid. In: Proc. of IEEE Ninth International Conference on Power Electronics and Drive Systems, 2011:424-429.

[3] Ibars C, Navarro M, Giupponi L. Distributed demand management in smart grid with a congestion game. In: Proc. of IEEE First Int'l Conference on Smart Grid Communications, 2010:495-500.

[4] Zhu Q, Han Z, Basar T. A differential game approach to distributed demand side management in smart grid. In: Proc. of IEEE International Conference on Communications, 2012:3345-3350.

[5] Miorandi D, De Pellegrini F. Demand-side management in smart grids: An evolutionary games perspective. In: Proc. of 6th International Conference on Performance Evaluation Methodologies and Tools, 2012:178-187.

[6] Mohsenian-Rad AH, Wong VWS, Jatskevich J, Schober R, Leon-Garcia A. Autonomous demand-side management based on game-theoretic energy consumption scheduling for the future smart grid. IEEE Transactions on Smart Grid, 2010;1(3):320331.

[7] Yaagoubi N, Mouftah HT. User-aware game theoretic approach for demand management. IEEE Transactions on Smart Grid, 2015;6(2)716-725.

[8] Gao J, Xiao Y, Liu J, Liang W, Chen CLP. A survey of communication/networking in Smart Grids. Future Gener. Comput. Syst, 2012;28(2):391-404.

[9] Vrba P, Marik V, Siano P, Leitao P, Zhabelova G, Vyatkin V, Strasser T. A review of agent and service-oriented concepts applied to intelligent energy systems. IEEE Transactions on Industrial Informatics, 2014;10(3):1890-1903.

[10] Cunjiang Y, Huaxun Z, Lei Z. Architecture design for smart grid. Energy Procedia, 2012;17(B):1524-1528.

[11] Fang X, Misra S, Xue G, Yang D. Smart grid-the new and improved power grid: A survey. IEEE Communications Surveys \& Tutorials, 2012;14(4):944-980.

[12] Babaioff M, Kleinberg R, Papadimitriou CH. Congestion games with malicious players. Games and Economic Behavior, 2009; 67(1):22-35.

[13] Saad W, Han Z, Poor HV, Basar T. Game-theoretic methods for the smart grid: An overview of microgrid systems, demandside management, and smart grid communications. IEEE Signal Processing Magazine, 2012;29(5):86-105.

[14] Fadlullah ZM, Nozaki Y, Takeuchi A, Kato N. A survey of game theoretic approaches in smart grid. In: Proc. of International Conference on Wireless Communications and Signal Processing, 2010:1-4.

[15] Samadi P, Mohsenian-Rad AH, Schober R, Wong VWS, Jatskevich J. Optimal real-time pricing algorithm based on utility maximization for smart grid. In: Proc. of First IEEE International Conference on Smart Grid Communications, 2010:415-420. 